\title{
Perception of tobacco, cannabis, and alcohol use of others is associated with one's own use
}

Nicolas Bertholet ${ }^{1 *}$, Mohamed Faouzi ${ }^{1}$, Joseph Studer ${ }^{1}$, Jean-Bernard Daeppen ${ }^{1}$ and Gerhard Gmel ${ }^{1,2,3,4}$

\begin{abstract}
Background: Interventions have been developed to reduce overestimations of substance use among others, especially for alcohol and among students. Nevertheless, there is a lack of knowledge on misperceptions of use for substances other than alcohol. We studied the prevalence of misperceptions of use for tobacco, cannabis, and alcohol and whether the perception of tobacco, cannabis, and alcohol use by others is associated with one's own use.

Methods: Participants $(n=5216)$ in a cohort study from a census of 20-year-old men $(N=11,819)$ estimated the prevalence of tobacco and cannabis use among peers of the same age and sex and the percentage of their peers drinking more alcohol than they did. Using the census data, we determined whether participants overestimated, accurately estimated, or underestimated substance use by others. Regression models were used to compare substance use by those who overestimated or underestimated peer substance with those who accurately estimated peer use. Other variables included in the analyses were the presence of close friends with alcohol or other drug problems and family history of substance use.
\end{abstract}

Results: Tobacco use by others was overestimated by $46.1 \%$ and accurately estimated by $37.3 \%$ of participants. Cannabis use by others was overestimated by $21.8 \%$ and accurately estimated by $31.6 \%$ of participants. Alcohol use by others was overestimated by more than half (53.4\%) of participants and accurately estimated by $31.0 \%$. In multivariable models, compared with participants who accurately estimated tobacco use by others, those who overestimated it reported smoking more cigarettes per week (incidence rate ratio [IRR] [95\% CI], 1.17 [range, 1.05, 1.32]). There was no difference in the number of cigarettes smoked per week between those underestimating and those accurately estimating tobacco use by others (IRR [95\% CI], 0.99 [range, 0.84, 1.17]). Compared with participants accurately estimating cannabis use by others, those who overestimated it reported more days of cannabis use per month (IRR [95\% CI], 1.43 [range, 1.21, 1.70]), whereas those who underestimated it reported fewer days of cannabis use per month (IRR [95\% Cl], 0.62 [range, 0.23, 0.75]). Compared with participants accurately estimating alcohol use by others, those who overestimated it reported consuming more drinks per week (IRR [95\% Cl], 1.57 [range, 1.43, 1.72]), whereas those who underestimated it reported consuming fewer drinks per week (IRR [95\% CI], 0.41 [range, 0.34, 0.50]).

Conclusions: Perceptions of substance use by others are associated with one's own use. In particular, overestimating use by others is frequent among young men and is associated with one's own greater consumption. This association is independent of the substance use environment, indicating that, even in the case of proximity to a heavy-usage group, perception of use by others may influence one's own use. If preventive interventions are to be based on normative feedback, and their aim is to reduce overestimations of use by others, then the prevalence of overestimation indicates that they may be of benefit to roughly half the population; or, in the case of cannabis, to as few as $20 \%$. Such interventions should take into account differing strengths of association across substances.

Keywords: Overestimation, Substance use, Perception, Alcohol, Tobacco, Cannabis

\footnotetext{
* Correspondence: Nicolas.Bertholet@chuv.ch

${ }^{1}$ Alcohol Treatment Center, Department of Community Medicine and Health, Centre Hospitalier Universitaire Vaudois, Beaumont 21b, P2, Lausanne, CHUV 1011, Switzerland

Full list of author information is available at the end of the article
}

\section{Biomed Central}

(c) 2013 Bertholet et al.; licensee BioMed Central Ltd. This is an open access article distributed under the terms of the Creative Commons Attribution License (http://creativecommons.org/licenses/by/2.0), which permits unrestricted use, distribution, and reproduction in any medium, provided the original work is properly cited. 


\section{Background}

According to social norms theory, our perceptions and beliefs about the "normal" behavior of others influences our own behavior [1]. For example, the belief that others drink alcohol, smoke tobacco, or use cannabis heavily is expected to influence the amount a person drinks alcohol, smokes tobacco, or uses cannabis [2]. Perceptions of how much alcohol others drink have been studied mostly among students [3-11]. Overestimating, or thinking that others drink more alcohol than oneself, is fairly prevalent and has been identified as a strong predictor of one's own alcohol use [11-14]. This misperception seems to apply to tobacco smoking [2,15], but less is known about whether it applies to other substances, such as cannabis $[15,16]$. Misperceptions have been observed among men and women; nevertheless, gender is a potential predictor of misperceptions of substance use by others. Evidence suggests that the magnitude of norm misperception is influenced by gender, and that men tend to overestimate norms to a lesser extent than women for alcohol and other substances [3,15].

Various interventions have been designed to address overestimations of use by others, with varying success, among college students [17-19]. These interventions aim at correcting misperceptions by providing normative feedback on prevailing alcohol and tobacco use norms. One hypothesis is that individuals with heavy use will decrease their consumption by correcting their overestimation of use by others. A review by Moreira et al. [20] provides evidence that normative feedback interventions for students have been effective in reducing alcohol use; brief interventions that include web-based interactive participation, where normative feedback is provided, also have had positive impacts on alcohol use and other alcohol-related outcomes. Many of these interventions are aimed at helping participants narrow the difference between perceived and actual behaviors of others (i.e., "correcting" the overestimation of use by others) $[1,20]$. In a recent test of the theoretical underpinnings of social norms theory, Johnson [21] showed that individuals whose perceptions of normative alcohol use became more accurate drank less alcohol.

Most of the substance use perceptions research has been conducted among students and focused on alcohol use. As such, there is a lack of knowledge on perception of tobacco use and cannabis use by others. Determining how frequently overestimations occur in the general population and how perceptions of use by others are associated with substance use (not limited to alcohol use) will yield information about perceptions as a potential factor to target in preventive interventions. Since both substance use and perceptions may be influenced by the behavior of close peers or family members, it is important to take into account whether or not individuals have been exposed to a heavy substance use environment [22].
Therefore, we studied the association between perceptions of tobacco, cannabis, and alcohol use by others with participants' own tobacco, cannabis, and alcohol use among 20-year-old Swiss men in the general population. We hypothesized that misperceptions of substance use by others are frequent and are associated with participants' current use.

\section{Methods}

The present study was part of the Cohort Study on Substance Use Risk Factors (C-SURF). Young Swiss men were approached for enrollment in this large cohort study when they presented at army recruitment centers in the French and German sectors of Switzerland, a country that has a mandatory two-day procedure to assess eligibility for military service. Virtually all 20-year-old Swiss men have to participate; thus, C-SURF participants were approached and recruited as they attended the centers at Lausanne (French sector) and Windisch (German sector). Even though Swiss men are approached for enrollment at the same age, some can attend the recruitment centers sooner than others: this explains the age range observed in studies conducted at the army recruitment centers by our group [13,23,24]. Switzerland is made up of 26 cantons (political subdivisions) and half-cantons. The Lausanne center processes conscripts from all of the French-speaking cantons (Berne [French-speaking parts], Fribourg, Genève, Jura, Neuchâtel, Valais, and Vaud), while the Windisch center processes conscripts from eight German-speaking cantons or half-cantons (Argau, Basel-Landschaft, Basel-Stadt, Luzern, Nidwalden, Obwalden, Solothurn, and Uri).

To minimize the risk of under- or over-reporting, participants were informed that all information they provided was confidential and had no implications for army conscription procedures. Participants were notified that the research was not connected to the army, and that military personnel could not see the responses or other data from any individual. Virtually all center attendees were eligible to participate in the study if they gave written informed consent. The Ethics Committee for Clinical Research at the Lausanne University Medical School approved the project.

During the C-SURF enrollment period (August 23, 2010 to July 31, 2011), 12,564 conscripts attended the designated army centers and were given the option of completing a brief self-administered screening questionnaire assessing alcohol and other drug use; 11,819 (94\%) completed it (considered hereafter as census data). All attendees were offered participation in the cohort study. Within two weeks after enrolment, attendees who gave consent were invited by mail or email to complete the cohort study questionnaire. Cohort study participants $(n=5216)$ completed a paper-and-pencil or online questionnaire containing items about substance use by others, which asked them to estimate the prevalence of peer tobacco and 
cannabis use and the percentage of peers who drink more alcohol than they do.

\section{Cohort study assessment Substance use}

The study questionnaire contained questions on drinking frequency (How often do you have a drink containing alcohol?) with answer choices of number of days per week (open-ended), 2-3 times a month, monthly or less, or never; and on alcohol quantity (How many drinks containing alcohol do you have on a typical day when you are drinking?) with a single open-ended answer (number of standard drinks). The time frame was the past 12 months. Number of standard drinks per week was obtained by multiplying the frequency and quantity questions. A standard drink was defined as $100 \mathrm{ml}$ of wine, $250 \mathrm{ml}$ of beer, $275 \mathrm{ml}$ of pre-mixed drink containing spirits, or $25 \mathrm{ml}$ of spirits (each containing about $10 \mathrm{~g}$ ethanol). Pictures of the drink equivalences accompanied each questionnaire.

Tobacco use was assessed with the following items: participants reporting any cigarette use over the past 12 months completed questions on tobacco frequency (How often, in general, have you smoked cigarettes in the past 12 months?) with answer choices of every day, 1-2, 3-4, or 5-6 days a week, 2-3 days per month, or once a month or less; and on tobacco quantity (On a usual day when you smoke cigarettes, how many cigarettes do you smoke?) with a single open-ended answer (number of cigarettes). The number of cigarettes smoked per week was obtained by multiplying frequency and quantity questions.

Cannabis use was assessed as follows: participants reporting any use by cannabis over the past 12 months completed a question on frequency (How often have you used cannabis over the past 12 months?) with answer choices of monthly or less, 2-4 times a month, 2-3 times a week, 4-5 times a week, or every day/almost every day.

\section{Perceptions}

Perceptions of smoking, cannabis, and alcohol use by others were assessed with the following items: What is the percentage of men your age that are smoking cigarettes? What is the percentage of men your age that are using cannabis? What is the percentage of men your age who are drinking more alcohol than you do?

\section{Additional variables}

Participants were asked whether or not they have family members (parents or siblings) with alcohol or drug problems. Family history was considered positive when at least one parent or sibling had an alcohol or drug problem. Participants were also asked the number of their close friends (none or one, two or some, or most) who have alcohol or drug problems, and were coded positive if they replied with some or most. The three choices were later collapsed into a dichotomous variable (none or one and two or some versus most) to indicate a heavy alcohol or drug use environment. Due to a strong association between presence of close friends with an alcohol problem and presence of close friends with a drug problem (chi-square $=1705.7,1 \mathrm{df}, \mathrm{p}<0.0001$ ), those two variables seemed to be carrying the same information; thus, a single variable (presence of close friends with alcohol or drug problems) was created. Education level was also reported.

\section{Substance use in the census \\ Prevalence of tobacco and cannabis use}

Census data from the short screening instrument that $94 \%$ of all conscripts completed was used to determine the prevalence of tobacco and cannabis use. To lessen the risk of exaggerated overestimations of prevalence of use in our study, we adopted a conservative attitude towards determining overestimations. Therefore, we chose the most inclusive definition of the prevalence of use in the census: in establishing the prevalence of tobacco and cannabis use, we considered those reporting any use of tobacco or cannabis over the past 12 months to be a user.

\section{Alcohol consumption}

Census norms were computed with the alcohol consumption data from the 11,819 individuals. The drinkingfrequency and alcohol-quantity questions were multiplied to obtain the number of standard drinks per week.

\section{Overestimation, underestimation and accurate estimation of substance use by others \\ Tobacco and cannabis use}

Overestimation, underestimation, and accurate estimation of the use of substances by others was determined by assessing how participants rated the prevalence of tobacco and cannabis use among peers of the same age and sex compared with the prevalence established in the census. A perceived prevalence within the $\pm 10 \%$ range of the prevalence established in the census was considered an accurate estimation of use by others. We a priori chose a $\pm 10 \%$ range for its clinical utility and to match the definitions found in other studies in the literature [15,25]. A smaller range could lead to a bias in favor of demonstrating that youth overestimate substance use by others. Proportions of conscripts overestimating, underestimating, and accurately estimating the prevalence of tobacco and cannabis use were determined.

\section{Alcohol use}

Perceived prevalence of alcohol use was not deemed relevant, given the actual high prevalence of alcohol use in 
the study population [24]. Therefore, estimations of alcohol use were made among alcohol users only, and each participant was asked to estimate how many individuals drank more alcohol than they did [13]. In order to determine for each person the proportion of the census that drank more alcohol than they did, weekly alcohol consumption (in standard drinks) by each of the study participants was compared with the weekly alcohol consumption reported by the census. The proportion of individuals in the census drinking more alcohol than a given study participant was compared with the perceived proportion reported by that participant. The prevalence of overestimation, underestimation, and accurate estimation of peer alcohol drinking was computed in the total sample. An accurate estimation was considered a perceived proportion within the $\pm 10 \%$ range of the computed proportion.

\section{Association between perception of use by others and substance use}

In a first step, separate over-dispersed Poisson regression models for tobacco, cannabis, and alcohol use were created to compare the substance use of those who overestimated, underestimated, and accurately estimated substance use by others. Those accurately estimating the use by others were used as the reference group. The "tobacco" model used the perception of tobacco use by others as the independent variable, the "cannabis" model used the perception of cannabis use by others as the independent variable, and the "alcohol" model used the perception of alcohol use by others as the independent variable. Outcome measures were number of cigarettes smoked per week for the "tobacco" model, number of days with cannabis use per month for the "cannabis" model, and number of standard drinks per week for the "alcohol" model. All three models were adjusted for age, education level, and linguistic sector. In a second step, presence of close friends with alcohol or drug problems and family history of alcohol or drug problems were added to the models, then adjusted for age, education level, and linguistic sector.

\section{Results}

Table 1 shows the characteristics of the 5216 participants. Nearly all (91.8\%) of the conscripts reported some alcohol use in the past 12 months; about half (47.5\%) used tobacco, and nearly a third (30.9\%) used cannabis. Among tobacco smokers, the mean (SD) number of cigarettes per week was 48.1 (54.8). Among cannabis users, the mean number of days per month using cannabis was 7.0 (9.2). For the alcohol users, the mean number of drinks per week was 8.4 (14.0). In the census $(\mathrm{n}=11,819)$, past-12-month prevalence was $44.5 \%$ for any tobacco use, $36.3 \%$ for any cannabis use, and $90.6 \%$ for any alcohol use.
Table 1 Participant characteristics

\begin{tabular}{lc}
\hline Participants characteristics ( $\mathbf{n}$ = 5216) & \\
\hline Age, mean (SD) & $19.5(1.3)$ \\
German speaking, $\mathrm{n}(\%)$ & $2097(40.2 \%)$ \\
Elementary education only, $\mathrm{n}(\%)$ & $2523(48.4 \%)$ \\
Any drinking, past 12 months, $\mathrm{n}(\%)$ & $4789(91.8 \%)$ \\
Mean number of drinks*/week among drinkers (SD) & $8.4(14.0)$ \\
Any tobacco use, past 12 months, $\mathrm{n}(\%)$ & $2478(47.5 \%)$ \\
Mean \# of cigarettes/week among users (SD) & $48.1(54.8)$ \\
Any cannabis use, past 12 months, $\mathrm{n}(\%)$ & $1612(30.9 \%)$ \\
Mean \# of days/month with cannabis among users (SD) & $7.0(9.2)$ \\
Close friends with alcohol or drug problems, ${ }^{* *} \mathrm{n}(\%)$ & $711(13.6 \%)$ \\
Family history of alcohol problems, & $388(7.4 \%)$ \\
Family history of drug problems, ${ }^{* * *} \mathrm{n}(\%)$ & $173(3.3 \%)$ \\
\hline
\end{tabular}

*Standard drink: $100 \mathrm{ml}$ of wine; $250 \mathrm{ml}$ of beer; $275 \mathrm{ml}$ of premixed drink containing spirits; or $25 \mathrm{ml}$ of spirits (each about $10 \mathrm{~g}$ of ethanol). **When participants reported that "some" or "most" of their close friends (versus "none" or "one or two") had an alcohol or drug problem, the variable was coded positively.

***We considered that a family history of alcohol or drug problems was present when participants reported at least one parent or sibling with an alcohol or drug problem.

\section{Perception of substance use by others}

The prevalence of tobacco use was overestimated by $46.1 \%$ and accurately estimated by $37.3 \%$ of study participants. The prevalence of cannabis use was overestimated by $21.8 \%$ and accurately estimated by $31.6 \%$ of the participants. When comparing their own alcohol use with alcohol use by others, more than half of participants (53.4\%) overestimated, while $31.0 \%$ accurately estimated. The proportion of participants overestimating, accurately estimating, and underestimating substance use is reported in Table 2.

Participants overestimating, accurately estimating, and underestimating tobacco use by others reported a mean (SD) of 25.4 (47.6), 20.9 (42.3), and 20.2 (41.8) cigarettes smoked per week, respectively. Participants overestimating, accurately estimating, and underestimating cannabis use by others reported a mean (SD) of 3.8 (7.9), 2.3 (6.2), and 1.3 (4.5) days of cannabis use per month, respectively. Participants overestimating, accurately estimating, and underestimating alcohol use by others reported a mean (SD) of 11.1 (16.0), 7.0 (12.4), and 2.9 (2.2) drinks per week, respectively.

\section{Associations between perception of use by others and substance use}

Separate over-dispersed Poisson regression models for tobacco, cannabis, and alcohol use were used to assess the associations between perception of use by others and substance use. All models were adjusted for age, education level, and linguistic sector. Results are expressed as incidence rate ratios (IRR) for the count variable in the model. 
Table 2 Overestimation, accurate estimation, and underestimation of others substance use

\begin{tabular}{llll}
\hline & Overestimation & Accurate estimation & Underestimation \\
\hline Tobacco* $^{*}$ & $46.1 \%$ & $37.3 \%$ & $16.6 \%$ \\
Cannabis* $^{*}$ & $21.8 \%$ & $31.6 \%$ & $46.6 \%$ \\
Alcohol** $^{*}$ & $53.4 \%$ & $31.0 \%$ & $15.6 \%$
\end{tabular}

*Using census data, we determined whether participants overestimated or underestimated the prevalence of tobacco or cannabis use by $10 \%$ or more. **The proportion of participants in the census drinking more than a given participant in the sample was compared with the perceived proportion reported by each of the participants of the study sample. The prevalence of overestimation, underestimation, and accurate estimation of peer drinking was computed for the total sample. An accurate estimation was considered a perceived proportion within the $\pm 10 \%$ range of the computed proportion.

The three count variables under study were number cigarette smoked per week, number of day of cannabis use per month, and number of standard alcohol drinks per week. The IRR gives the factor change in the expected count compared with the reference category. For example, if participants with characteristic $\mathrm{X}$ are compared with the reference group without the characteristic $\mathrm{X}$ with respect to their weekly alcohol use, an IRR $=1$ means that, holding all other variables constant, having the characteristic $\mathrm{X}$ does not change the expected number of drinks per week. An IRR $=0.8$ means that, on average, the group with the characteristic X reports $20 \%$ less drinks per week than the group without the characteristic $\mathrm{X}$ and an IRR $=2$ means that, on average, the group with the characteristic $\mathrm{X}$ reports twice the number of drinks per week than the group without the characteristic X.

\section{Tobacco use}

Compared with participants accurately estimating tobacco use by others, those who overestimated it reported more cigarettes smoked per week (incidence rate ratio (IRR) [95\% CI], 1.19 [range, 1.06, 1.34]). There was no difference in the number of cigarettes smoked per week between those underestimating and those accurately estimating tobacco use by others (IRR [95\% CI], 0.97 [range, 0.82, 1.15]).

\section{Cannabis use}

Compared with participants accurately estimating cannabis use by others, those who overestimated it reported more days of cannabis use per month (IRR [95\% CI], 1.59 [range, $1.33,1.89]$ ), whereas those who underestimated it reported less days of cannabis use per month (IRR [95\% CI], 0.57 [range, 0.47, 0.69]).

\section{Alcohol use}

Compared with participants accurately estimating alcohol use by others, those who overestimated it reported consuming more drinks per week (IRR [95\% CI], 1.57 [range, 1.42, 1.74]), whereas those who underestimated it reported consuming fewer drinks per week (IRR [95\% CI], 0.41 [range, $0.33,0.50$ ]).

Table 3 shows the results of the same three models after adding presence of close friends with an alcohol or drug problem, family history of alcohol problems, and family history of drug problems. Independent associations of perceptions with substance use remained. Compared with participants accurately estimating tobacco use by others, those who overestimated it reported more cigarettes smoked per week (IRR [95\% CI], 1.17 [range, 1.05, 1.32]). There was no difference in the number of cigarettes smoked per week between those underestimating and those accurately estimating tobacco use by others (IRR [95\% CI], 0.99 [range, 0.84, 1.17]). Compared with participants accurately estimating cannabis use by others, those who overestimated it reported more days of cannabis use per month (IRR [95\% CI], 1.43 [range, 1.21, 1.70]), while those who underestimated it reported fewer days of cannabis use per month (IRR [95\% CI], 0.62 [range, 0.23, 0.75]). Compared with participants accurately estimating alcohol use by others, those who overestimated it reported more drinks consumed per week (IRR [95\% CI], 1.57 [range, 1.43, 1.72]), while those who underestimated it reported fewer drinks consumed per week (IRR [95\% CI], 0.41 [range, 0.34, $0.50]$ ). There were the following significant positive associations: having close friends with alcohol or drug problems and one's own tobacco, cannabis, and/or alcohol use; family history of alcohol problems and one's own tobacco and/or alcohol use; and family history of drug problems and one's own tobacco, alcohol, and/or cannabis use.

\section{Discussion}

Perceptions of substance use by others are associated with one's own use among young men; specifically, our results show that overestimating substance use by others is associated with greater consumption. In addition, underestimating the substance use by others appears associated with less use, except for tobacco. Our study adds important information about the frequency of overestimation, underestimation, and accurate estimation of substance use and the association of overestimation of use by others with current use, especially for tobacco and cannabis use where evidence has been scarce $[2,16,26]$.

The magnitude of the associations between perceptions and usage was similar in the models where variables, such as having close friends with alcohol or drug problems or having a family history of alcohol or drug problems, are added or taken out. The relationship most affected by the addition of these variables in multivariable models was overestimation of cannabis use by others and participants' own cannabis use (IRR 1.59 versus 1.43 in the model containing the close friends with alcohol or drug problems and family history variables). These results are in line with the literature $[11,13,14,16]$. The magnitude of the observed 
Table 3 Multivariable models examining the association of substance use perception, presence of close friends with alcohol or drug problems, and family history of alcohol or drug problems with substance use

\begin{tabular}{|c|c|}
\hline Model 1: tobacco use* & Cigarettes/week, IRR (95\% Cl) \\
\hline \multicolumn{2}{|c|}{$\begin{array}{l}\text { Perception of tobacco use of others (same age/sex), } \\
\text { reference group = accurate estimation of tobacco use of others }\end{array}$} \\
\hline Overestimation of tobacco use by others & $1.17(1.05 ; 1.32)$ \\
\hline Underestimation of tobacco use by others & $0.99(0.84 ; 1.17)$ \\
\hline Close friends with alcohol or drug problems & $2.08(1.83 ; 2.35)$ \\
\hline Family history of alcohol problems (parents/siblings) & $1.48(1.25 ; 1.75)$ \\
\hline Family history of drug problems (parents/siblings) & $1.52(1.22 ; 1.89)$ \\
\hline Model 2: cannabis use* & Days of cannabis use/month, IRR $(95 \% \mathrm{Cl})$ \\
\hline \multicolumn{2}{|c|}{$\begin{array}{l}\text { Perception of cannabis use of others (same age/sex), } \\
\text { reference group = accurate estimation of cannabis use of others }\end{array}$} \\
\hline Overestimation of cannabis use by others & $1.43(1.21 ; 1.70)$ \\
\hline Underestimation of cannabis use by others & $0.62(0.23 ; 0.75)$ \\
\hline Close friends with alcohol or drug problems & $2.91(2.48 ; 3.42)$ \\
\hline Family history of alcohol problems (parents/siblings) & $1.12(0.89 ; 1.42)$ \\
\hline Family history of drug problems (parents/siblings) & $1.78(1.36 ; 2.33)$ \\
\hline Model 3: drinking* & Drinks/week, IRR (95\% CI) \\
\hline \multicolumn{2}{|l|}{$\begin{array}{l}\text { Perception of drinking of others (same age/sex), } \\
\text { reference group = accurate estimation of drinking of others }\end{array}$} \\
\hline Overestimation of drinking of others & $1.57(1.43 ; 1.72)$ \\
\hline Underestimation of drinking of others & $0.41(0.34 ; 0.50)$ \\
\hline Close friends with alcohol or drug problems & $1.43(1.28 ; 1.59)$ \\
\hline Family history of alcohol problems (parents/siblings) & $1.35(1.17 ; 1.55)$ \\
\hline Family history of drug problems (parents/siblings) & $1.38(1.14 ; 1.67)$ \\
\hline
\end{tabular}

${ }^{*}$ All models were adjusted for age, education level, and linguistic region. IRR = incidence rate ratio.

associations between perceptions of use by others and substance use are of clinical significance: compared with participants accurately estimating alcohol use by others, participants who overestimated alcohol use by others reported drinking almost $60 \%$ more per week. Those underestimating alcohol use by others reported drinking $60 \%$ less per week than those accurately estimating. For cannabis, these differences were also of clinical significance and were in the $40 \%$ range. Though smaller, the association between perception of tobacco use by others and tobacco use was still clinically significant: participants overestimating tobacco use by others smoked $17 \%$ more cigarettes per week compared with those accurately estimating it.

As a potential target of normative feedback interventions, overestimations of use by others have been the focus of various research studies. Our results show that overestimations of substance use by others are frequent among young men (but vary by substance) and are associated with greater consumption. These associations are independent of being in a heavy alcohol or drug using environment, indicating that, even in the case of proximity to these groups, overestimations of use by others may influence one's own use. Our results show that the overestimation of use by others has a stronger association with one's own use for cannabis and alcohol than it does for tobacco. This suggests a potentially differential influence of perceptions on one's own use of these substances. An alternate explanation for overestimations is that individuals select their friends and acquaintances based on shared preferences in alcohol, tobacco, and drug use, and overestimations of behaviors within a "distal" group of the same of peers of same age and sex may reflect peer usage more than anything else may. Using "distal" groups in research on substance use perceptions (i.e., individuals of the same age and gender as opposed to more "proximal" groups such as friends or people in the same fraternity/sorority) has faced criticism and has been presented as a source of exaggerated overestimations [27]. We attempted to take into account the influence of a heavy using environment and heritability by showing independent associations of one's own use and overestimations of others' use with presence of close friends and family members with alcohol and drug problems. Even though misperceptions of a distal group are less likely to have an influence on behavior than misperceptions of a proximal group, we still observed associations between overestimations and substance use [28]. 
We observed strong variations in the frequency of overestimation by substance: overestimation of alcohol and tobacco use was observed in more than $45 \%$ of the participants, while overestimation of cannabis use was observed in $22 \%$ of participants. We do not have a straightforward explanation for the lower rates of overestimation of cannabis use. One possible explanation is that cannabis may have received less attention than tobacco and alcohol, or its consumption may be less visible due to its illegal status in Switzerland. Determining the causes of these variations is an area for future research.

The association of secondary variables (close friends with alcohol or drug problems, and family substance use history) with participants' own substance use is concordant with research showing that environmental [29-31] and genetic influences [32] play a role in individual's substance use [22]. In the present study, both family history of drug or alcohol problems and markers of peer behavior were associated with substance use. Family history can be seen both as a marker of genetic and environmental influences, since heritability and parental and family-member modeling of substance use behaviors can be associated with substance use $[22,32,33]$. Generally, family history associations were found across substances: family history of drug problems was associated with alcohol, tobacco, and cannabis use, and family history of alcohol problems was associated with alcohol and tobacco use. The presence of close friends with alcohol or drug problems (a proxy for a heavy using environment) was also associated with substance use.

This study has several limitations. First, it should be noted that we relied on self-reported measures for substance use and other factors. Notably, participants were asked to report whether or not they had family members or friends with alcohol and or drug problems, and the psychometric properties of these questions were not tested. Second, we assessed only descriptive norms, and interpersonal perception was limited to those of the same age and sex as the participant. Perception of the amount of alcohol used by others in more proximal groups (such as close friends) was not evaluated. The large number of participants in the study and the logistics of the screening survey preclude gathering detailed data that could be obtained during face-to-face structured interviews. In addition, we a priori chose a $\pm 10 \%$ range to define "accurate perception." A smaller range could have introduced a bias towards demonstrating that youth overestimate substance use by others; however, it should be noted that this definition impacts the prevalence of over-, under-, and accurate estimations. Sensitivity analyses around this criterion were performed (using $\mathrm{a} \pm 5 \%$ and $\mathrm{a} \pm 20 \%$ range). We did not observe major differences in the associations reported herein. Third, because it is a cross-sectional design, we were not able to investigate possible causal pathways or assess the influence of changes in perception on substance use. In addition, only 20-year-old men were included, and our results cannot be generalized beyond this population subset. Evidence suggests that the magnitude of norm misperception is influenced by gender. Even though misperceptions have been observed across gender, their magnitude is likely to differ. Therefore, our results should not be extrapolated to women. Fourth, about $6 \%$ of the invited conscripts refused to complete the screening questionnaire; therefore, we cannot be certain that alcohol, tobacco, and cannabis use was accurately measured in the census. Nevertheless, it is unlikely that this unduly affected measures of substance use prevalence in the census or significantly influenced the obtained perceptions, since we were able to rely on screening data from $94 \%$ of the census. Cohort participation and response to cohort questionnaires may be subject to self-selection bias. Although minimally, participants who did complete the cohort questionnaire differed from the source population. Substance use was lower among those who completed the cohort questionnaire compared with census data. The non-response bias was $<10 \%$ for alcohol and cannabis and $<15 \%$ for tobacco use. Detailed analyses are presented elsewhere [34,35].

In 2012, Hilde Pape pointed out methodological limitations in studies focusing on estimations of peer substance use and argued that these restrictions may lead to exaggerated results [27]. She reported that the representativeness of studies in the field is problematic because of convenience samples and low response rates. In this regard, our study has the advantage of using a large sample with perception items imbedded within a larger cohort questionnaire. Also, because $94 \%$ of the source population was assessed on substance use, the representativeness of our cohorts has been well characterized. Even if differences existed between cohort participants and the source population, these differences were limited [34]. Other methodological concerns reported by Pape include the complexity of questions used to assess perceptions. Particularly for the item on alcohol use perception, this limitation exists in the present study. Asking participants to compare their alcohol use to alcohol use by others by having them determine the proportion of individuals their age and sex who drink more alcohol than they do can be challenging, and assumes pre-existing beliefs about the use of others. Questions on tobacco and cannabis use perceptions were less complex, even though they also assumed pre-existing beliefs and did not include "I don't know" as a choice. There is no way to determine the level or strength of pre-existing beliefs in an individual. Therefore, the present study is also limited by the complexity of the questions used to assess perceptions.

Nevertheless, we believe our study has some notable strengths. We were able to assess actual and perceived substance use in samples from the same population that 
included consumption screening data from $94 \%$ of the census and from a large sample of cohorts. We took into account proxies of peer use in the adjusted analyses, which allowed an assessment of independent associations between perceptions of use and one's own use. Even though interpersonal perceptions were limited to peers of the same age and sex, the fact that we assessed family history and the presence of close friends with substance use problems strongly supports the hypothesis of an independent association of perceived substance use by others with one's current own use. Also, we used norms that are usually used in available normative feedback interventions (i.e., in currently available interventions, notably web-based normative-feedback interventions, substance use by a given individual is compared with the substance use of a population of the same age and sex, and not to the consumption of close friends, which would be challenging to determine).

\section{Conclusion}

Our results confirm among young men in the general population what has already been seen in selected student populations; i.e., overestimations of substance use occur frequently and are associated with greater usage. Although we cannot make a causal interpretation because of our study design, our results suggest that perceptions themselves may influence current behavior, and that those who overestimate substance use by others are indeed likely to use more themselves. We believe our study adds information on the prevalence of misperceptions for substances other than alcohol. It is important to note that $30-40 \%$ of the participants accurately estimated use by others, and that a substantial proportion underestimated use by others.

If preventive interventions are to be based on normative feedback and their aim is to reduce misperceptions, then the prevalence of overestimation indicates that they may benefit about half of the population, or in the case of cannabis, as little as about $20 \%$ of the population. To be successful, such interventions should take into account differential strengths of association across substances, especially if large-scale efforts to implement interventions based primarily on this theoretical rationale are attempted.

\section{Competing interests}

The authors declare that they have no competing interests.

\section{Authors' contributions}

NB designed the study, analyzed the data, and wrote the initial draft and final version of the article. JS participated in planning and data analyses, discussed earlier versions, and reviewed the final version of the article. MF participated in planning and data analyses, reviewed the analyses, discussed earlier versions, and reviewed the final version of the article. J-BD designed the study discussed earlier versions and reviewed the final version of the article. GG designed the study, discussed analyses, discussed earlier versions and reviewed the final version of the article. All authors contributed to and approved the final manuscript.

\section{Acknowledgments}

We are grateful to Charlotte Eidenbenz for her extensive efforts to coordinate the study. We thank George Danko for the careful editing of the manuscript.

\section{Funding}

The study was funded by the Swiss National Science Foundation (Cohort Study on Substance Use Risk Factors, 33CSC0-122679).

\section{Author details}

'Alcohol Treatment Center, Department of Community Medicine and Health, Centre Hospitalier Universitaire Vaudois, Beaumont 21 b, P2, Lausanne, CHUV 1011, Switzerland. ${ }^{2}$ Swiss Institute for the Prevention of Alcohol and Drug Problems, Lausanne, Switzerland. ${ }^{3}$ Centre for Addiction and Mental Health, Toronto, Ontario, Canada. ${ }^{4}$ University of the West of England, Bristol, UK.

Received: 17 May 2013 Accepted: 10 October 2013

Published: 19 October 2013

\section{References}

1. Berkowitz AD: An overview of the social norms approach. In Changing the culture of college drinking: a socially situated health communication campaign. Edited by Lederman L, Stewart L. Creskill, NJ: Hampton Press; 2005.

2. Cunningham JA, Selby PL: Implications of the normative fallacy in young adult smokers aged 19-24 years. Am J Public Health 2007, 97:1399-1400.

3. Borsari B, Carey KB: Descriptive and injunctive norms in college drinking: a meta-analytic integration. J Stud Alcohol 2003, 64:331-341.

4. Carey KB, Borsari B, Carey MP, Maisto SA: Patterns and importance of self-other differences in college drinking norms. Psychol Addict Behav 2006, 20:385-393.

5. Borsari B, Carey KB: Peer influences on college drinking: a review of the research. J Subst Abuse 2001, 13:391-424.

6. Borsari $B E$, Carey $K B$ : Understanding fraternity drinking: five recurring themes in the literature, 1980-1998. J Am Coll Health 1999, 48:30-37.

7. Perkins HW, Haines MP, Rice R: Misperceiving the college drinking norm and related problems: a nationwide study of exposure to prevention information, perceived norms and student alcohol misuse. J Stud Alcohol 2005:470-478.

8. Kypri K, Langley JD: Perceived social norms and their relation to university student drinking. J Stud Alcohol 2003, 64:8.

9. Werch CE, Pappas DM, Carlson JM, DiClemente CC, Chally PS, Sinder JA: Results of a social norm intervention to prevent binge drinking among first-year residential college students. J Am Coll Health 2000, 49:85-92.

10. França $L R$, Dautzenberg $B$, Reynaud M: Heavy episodic drinking and alcohol consumption in French colleges: the role of perceived social norms. Alcohol Clin Exp Res 2010, 34:1-11.

11. McAlaney J, McMahon J: Normative beliefs, misperceptions, and heavy episodic drinking in a British student sample. J Stud Alcohol Drugs 2007, 68:385-392.

12. Perkins HW, Wechsler H: Variation in perceived college drinking norms and its impact on alcohol abuse: a nationwide study. J Drug Issues 1996, 26:961-974.

13. Bertholet N, Gaume J, Faouzi M, Daeppen JB, Gmel G: Perception of the amount of drinking by others in a sample of 20-year-old men: the more I think you drink, the more I drink. Alcohol Alcohol 2011, 46:83-87.

14. Lewis MA, Litt DM, Blayney JA, Lostutter TW, Granato H, Kilmer JR, Lee CM: They drink how much and where? Normative perceptions by drinking contexts and their association to college students' alcohol consumption. J Stud Alcohol Drugs 2011, 72:844-853.

15. Riou Franca L, Dautzenberg B, Falissard B, Reynaud M: Peer substance use overestimation among French university students: a cross-sectional survey. BMC Public Health 2010, 10:169.

16. Kilmer JR, Walker DD, Lee CM, Palmer RS, Mallett KA, Fabiano P, Larimer ME: Misperceptions of college student marijuana use: implications for prevention. J Stud Alcohol 2006, 67:277-281.

17. Kypri K, Saunders JB, Williams SM, McGee RO, Langley JD, Cashell-Smith ML Gallagher SJ: Web-based screening and brief intervention for hazardous drinking: a double-blind randomized controlled trial. Addiction 2004 99:1410-1417.

18. Walters ST, Vader AM, Harris TR: A controlled trial of web-based feedback for heavy drinking college students. Prev Sci 2007, 8:83-88. 
19. Carey KB, Carey MP, Maisto SA, Henson JM: Brief motivational intervention for heavy college drinkers: a randomized controlled trial. J Consult Clin Psychol 2006, 74:943-954.

20. Moreira MT, Smith LA, Foxcroft D: Social norms interventions to reduce alcohol misuse in university or college students. Cochrane Database Syst Rev 2009, 3, CD006748.

21. Johnson MB: Experimental test of social norms theory in a real-world drinking environment. J Stud Alcohol Drugs 2012, 73:851-859.

22. Stone AL, Becker LG, Huber AM, Catalano RF: Review of risk and protective factors of substance use and problem use in emerging adulthood. Addict Behav 2012, 37:747-775.

23. Daeppen JB, Bertholet N, Gaume J, Fortini C, Faouzi M, Gmel G: Efficacy of brief motivational intervention in reducing binge drinking in young men: a randomized controlled trial. Drug Alc Depend 2011, 113:69-75.

24. Gmel G, Gaume J, Faouzi M, Kulling JP, Daeppen JB: Who drinks most of the total alcohol in young men-risky single occasion drinking as normative behaviour. Alcohol Alcohol 2008, 43:692-697.

25. Wechsler $\mathrm{H}$, Kuo M: College students define binge drinking and estimate its prevalence: results of a national survey. J Am Coll Health 2000, 49:57-64.

26. Wolfson S: Students' estimates of the prevalence of drug use: evidence for a false consensus effect. Psychol Addict Behav 2000, 14:295-298.

27. Pape $\mathrm{H}$ : Young people's overestimation of peer substance use: an exaggerated phenomenon? Addiction 2012, 107:878-884

28. Yanovitzky I, Stewart LP, Lederman LC: Social distance, perceived drinking by peers, and alcohol use by college students. Health Commun 2006, 19:1-10.

29. Larsen H, Overbeek G, Granic I, Engels RC: The strong effect of other people's drinking: two experimental observational studies in a real bar. Am J Addict 2012, 21:168-175.

30. Weitzman ER, Nelson TF, Wechsler $\mathrm{H}$ : Taking up binge drinking in college: the influences of person, social group, and environment. J Adolesc Health 2003, 32:26-35.

31. Stoddard SA, Bauermeister JA, Gordon-Messer D, Johns M, Zimmerman MA: Permissive norms and young adults' alcohol and marijuana use: the role of online communities. J Stud Alcohol Drugs 2012, 73:968-975.

32. Schuckit MA: An overview of genetic influences in alcoholism. J Subst Abuse Treat 2009, 36:S5-S14.

33. Hawkins JD, Catalano RF, Miller JY: Risk and protective factors for alcohol and other drug problems in adolescence and early adulthood: implications for substance abuse prevention. Psychol Bull 1992, 112:64-105.

34. Studer J, Baggio S, Mohler-Kuo M, Dermota P, Gaume J, Bertholet N, Daeppen JB, Gmel G: Examining non-response bias in substance use research: are late respondents proxies for non-respondents? Drug Alcohol Depend 2013, 132:316-323.

35. Studer J, Mohler-Kuo M, Dermota P, Gaume J, Bertholet N, Eidenbenz C, Daeppen JB, Gmel G: Need for informed consent in substance use studies: harm of bias? J Stud Alcohol Drugs. in press.

Cite this article as: Bertholet et al:: Perception of tobacco, cannabis, and alcohol use of others is associated with one's own use. Addiction Science \& Clinical Practice 2013 8:15.

\section{Submit your next manuscript to BioMed Central and take full advantage of:}

- Convenient online submission

- Thorough peer review

- No space constraints or color figure charges

- Immediate publication on acceptance

- Inclusion in PubMed, CAS, Scopus and Google Scholar

- Research which is freely available for redistribution 